\title{
Josephson charge-phase qubit with radio frequency readout: coupling and decoherence
}

\author{
A. B. Zorin* \\ Physikalisch-Technische Bundesanstalt, Bundesallee 100, 38116 Braunschweig, Germany
}

(Dated: 9 December, 2003)

\begin{abstract}
The Josephson charge-phase qubit based on a superconducting single charge transistor inserted in a low-inductance superconducting loop is considered. The loop is inductively coupled to a radiofrequency driven tank circuit enabling the readout of the qubit states by measuring the effective Josephson inductance of the transistor. The effect of qubit dephasing and relaxation due to electric and magnetic control lines as well as the measuring system is evaluated. Recommendations for the qubit operation in the magic points with minimum decoherence are given.
\end{abstract}

PACS numbers: 74.50.+r, 85.25.Cp, 03.67.Lx

\section{INTRODUCTION}

The superconducting quantum bit (qubit) circuits comprising mesoscopic Josephson tunnel junctions have recently demonstrated remarkable quantum coherence properties and now are considered to be promising elements for a scalable quantum computer [1]. The readout of macroscopic quantum states of a single qubit or a system of coupled qubits with minimum decoherence being caused by the detector, remains, however, one of the most important engineering issues in this field.

The Josephson qubits are commonly subdivided into flux, phase, charge and charge-phase qubits. The design of charge and charge-phase qubits is based on a Cooper pair box 2] in which a small superconducting island with significant Coulomb energy is charged through a small Josephson junction (charge qubit) or a miniature doublejunction SQUID (charge-phase). The distinct quantum states of the box generated by signals applied to a gate are associated with different observable charges on the island. This makes it possible to read out the qubit state by discriminating the island charge. Probing this charge can be done either by single quasiparticle tunneling across a small auxiliary tunnel junction attached to the island 3] or by a capacitively coupled electrometer 4]. In the charge-phase qubits, the quantum states of the box involve the phase coordinate of the SQUID loop, so discriminating these states can also be done by measuring the persistent current circulating in the loop at an appropriate dc flux bias. Such a measurement was performed in the experiment of the Saclay group [5]. In their setup, nicknamed "Quantronium", the circulating current passed through a larger auxiliary (third) junction was read out by measuring the switching current of this junction.

The persistent current is not, however, the only phasedependent quantity characterizing the quantum state of the charge-phase qubit. Another useful quantity is the Josephson inductance of the double junction, which can be probed by small rf oscillations induced in the qubit. Recently, we proposed a transistor configuration of the
Cooper pair box (see Fig.1), in which the macroscopic superconducting loop closing the transistor terminals was inductively coupled to a radio-frequency tank circuit [6]. Similar to the rf-SQUID-based method of measurement of the Josephson junction impedance [7], this setup makes it possible to measure the rf impedance (more specifically, the Josephson inductance) of the system of two small tunnel junctions connected in series and in doing so, to probe the macroscopic states of the qubit.

On the one hand, the advantage of this method con-

(a)

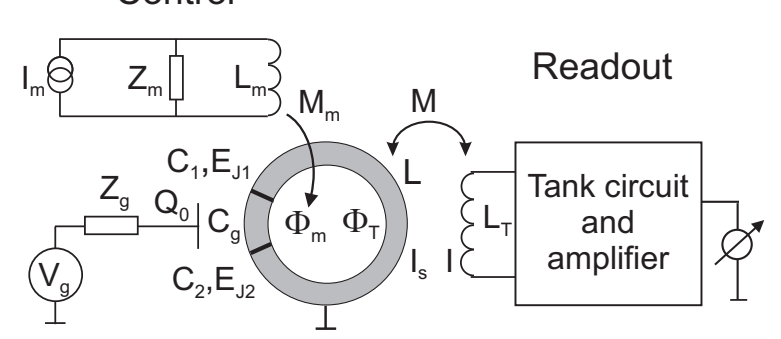

(b)

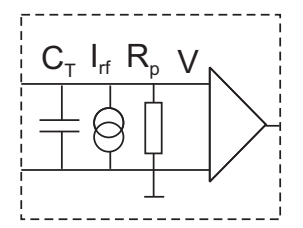

(c)

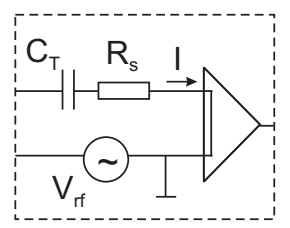

FIG. 1: (a) The electric circuit diagram of the charge-flux qubit inductively coupled to a tank circuit by mutual inductance $M$. The macroscopic superconducting loop of inductance $L$ is interrupted by two small Josephson tunnel junctions positioned close to each other and forming a single charge transistor; the capacitively coupled gate polarizes the island of this transistor. The qubit is controlled by charge $Q_{0}$ generated by the gate and flux $\Phi_{m}$ induced by coil $L_{m}$. The tank circuit which is either of a parallel (b) or a serial (c) type is driven by a harmonic signal ( $I_{\mathrm{rf}}$ or $V_{\mathrm{rf}}$, respectively) of frequency $\omega_{\text {rf }} \approx \omega_{0}$, the resonant frequency of the uncoupled tank circuit. 
sists in an effective decoupling between the qubit and a measurement device, that reduces the decoherence of the qubit. Moreover, the loop design of the qubit has a potential to perform a data readout in a non-destructive way [8]. On the other hand, due to the selective characteristic of the tank, the bandwidth of this setup is rather narrow, so the optimum relation between the relaxation time of the qubit and the time of measurement becomes an issue. Furthermore, the driving rf signal may induce appreciable frequency modulation and dephasing of the qubit during its evolution (performance of the quantum operations). Switching the oscillations off and on is, however, possible only on a relatively long-time scale of a transient process in the tank.

In this paper we address the problem of decoherence induced in the charge-flux qubit by the classical resonance tank circuit. Besides, we propose a strategy of measurement and optimize the regime of qubit operation for typical parameters of the circuit.

\section{BACKGROUND}

The small tunnel junctions of the charge-flux qubit are characterized by self-capacitances $C_{1}$ and $C_{2}$ and the Josephson coupling strengths $E_{J 1}$ and $E_{J 2}$. These junctions with a small central island in-between and a capacitively coupled gate therefore form a single charge transistor connected in our network as the Cooper pair box (see Fig.1). Critical currents of the junctions are equal to $I_{c 1, c 2}=\frac{2 \pi}{\Phi_{0}} E_{J 1, J 2}$, where $\Phi_{0}=h / 2 e$ is the flux quantum, and their mean value $I_{c 0}=\frac{1}{2}\left(I_{c 1}+I_{c 2}\right)$. The design enables magnetic control of the Josephson coupling in the box in a dc SQUID manner. The system therefore has two parameters, the total Josephson phase across the two junctions $\phi=\varphi_{1}+\varphi_{2}=2 \pi \Phi / \Phi_{0}$ controlled by flux $\Phi$ threading the loop and the gate charge $Q_{0}$ set by the gate voltage $V_{g}$. The geometrical inductance of loop $L$ is assumed to be much smaller than the Josephson inductance of the junctions $L_{J 0}=\Phi_{0} /\left(2 \pi I_{c 0}\right)$,

$$
\beta_{L}=L / L_{J 0} \ll 1
$$

Neglecting the magnetic energy term associated with the current through the small inductance $L$, the Hamiltonian of the autonomous qubit circuit is expressed as

$$
H_{0}=\frac{\left(2 e n-Q_{0}\right)^{2}}{2 C}-E_{J}(\phi) \cos \chi .
$$

The second term in Eq. (2) originates from the total Josephson energy equal to $-E_{J 1} \cos \varphi_{1}-E_{J 2} \cos \varphi_{2}$. The effective Josephson coupling strength is

$$
E_{J}(\phi)=\left(E_{J 1}^{2}+E_{J 2}^{2}+2 E_{J 1} E_{J 2} \cos \phi\right)^{1 / 2},
$$

$\left|E_{J 1}-E_{J 2}\right| \leq E_{J}(\phi) \leq E_{J 1}+E_{J 2} \equiv 2 E_{J 0}=\frac{\Phi_{0}}{\pi} I_{c 0}$, while a phase variable $\chi=\varphi+\gamma(\phi)$. The angle $\gamma$ is given by the expression

$$
\tan \gamma=\left(j_{1}-j_{2}\right) \tan (\phi / 2)
$$

where dimensionless Josephson energies $j_{1,2}=$ $E_{J 1, J 2} /\left(2 E_{J 0}\right)$ with $j_{1}+j_{2}=1$. The difference phase $\varphi=\frac{1}{2}\left(\varphi_{1}-\varphi_{2}\right)$ is a variable conjugate to the island charge $2 e n=-2 e i \frac{\partial}{\partial \varphi}$ and $n$ is the operator of the number of excess Cooper pairs on the island. This charge enters the charging energy (first) term in Eq. (2), in which $C$ is the total capacitance of the island, $C=C_{1}+C_{2}+C_{g} \approx C_{1}+C_{2}$ and the gate capacitance $C_{g} \ll C_{1,2}$. The characteristic charging energy, $E_{c}=e^{2} / 2 C$ is assumed to be of the order of the Josephson coupling energies $E_{J 1} \sim E_{J 2} \gg k_{B} T$.

The Schrödinger equation corresponding to the Hamiltonian Eq. (2) is the Mathieu's equation [9]. The eigen energies form the Bloch bands and eigenfunctions $|n, q\rangle$ are the Bloch wave functions of a particle in the periodic (Josephson) potential with "quasimomentum" (here rather quasicharge) $q$. Its value is the charge which the gate source provides to the island, i.e., $q=Q_{0}=C_{g} V_{g}$. Each of such eigen functions can be presented as a coherent superposition of the plain waves,

$$
|q, n\rangle=\sum_{m} C_{m}^{(n)} \exp \left[i\left(\frac{q}{2 e}+m\right) \chi\right],
$$

where $m=0, \pm 1, \pm 2, \ldots$ is the number of excess Cooper pairs on the island 10,11]. The weights of these coherent contributions $\left|C_{m}^{(n)}\right|^{2}$ depend on $q$, the band index $n$ and the characteristic ratio

$$
\lambda=E_{J}(\phi) / E_{c}
$$

The two lowest energy levels $E_{n}(q, \phi)$, i.e., $n=0$ and 1 (see their dependencies on $q$ and $\phi$ in Fig.2), form the basis $\{|0\rangle,|1\rangle\}$ suitable for qubit operation. In this basis, the Hamiltonian (2) is diagonal,

$$
H_{0}=\frac{1}{2} \epsilon \sigma_{z}
$$

where $\sigma_{i}$ with $i=x, y, z$ is Pauli spin operator. The general state of qubit is

$$
|\Psi\rangle=a|0\rangle+b|1\rangle
$$

with $|a|^{2}+|b|^{2}=1$. It is remarkable that the level spacing $\epsilon(q, \phi) \equiv \hbar \Omega=E_{1}(q, \phi)-E_{0}(q, \phi)$ and, therefore, the transition frequency $\Omega$ are efficiently controlled by two knobs, i.e., by varying the parameters $q$ and $\phi$ (or, equivalently, $Q_{0}$ and $\left.\Phi\right)$ [12].

The idea underlying the measurement of this chargeflux qubit is based on inducing radio frequency oscillations in the tank circuit of frequency $\omega_{\text {rf }} \ll \Omega[6]$. Due to 
inductive coupling $M$, these oscillations cause oscillations of corresponding flux $\Phi_{T}$ (see Fig. 1a) and, therefore, of total phase,

$$
\phi=\frac{2 \pi}{\Phi_{0}}\left(\Phi_{T}+\Phi_{m}\right)=\phi_{a} \sin \left(\omega_{\mathrm{rf}} t+\theta\right)+\phi_{0} .
$$

If the rf drive signal is sufficiently weak, the amplitude $\phi_{a}$ of these oscillations is relatively small, $\phi_{a} \ll \pi$. In this linear regime the reverse Josephson inductance is equal to

$$
L_{J}^{-1}(n, q, \phi)=\left(\frac{2 \pi}{\Phi_{0}}\right)^{2} \frac{\partial^{2} E_{n}(q, \phi)}{\partial \phi^{2}},
$$

i.e. it is determined by the local curvature of the energy surface $E_{n}$. For example, for $E_{J 0}=2 E_{c}$ (see Fig. 2) at $q \approx 0$, we have the following estimates within the zero and first bands:

$$
L_{J}^{-1}(0,0, \phi) \approx 0.4 L_{J 0}^{-1} \cos \phi
$$

and

$$
L_{J}^{-1}(1,0, \phi) \approx 0.1 L_{J 0}^{-1} \cos \phi,
$$

respectively. In the vicinity of the level avoided crossing point, $q=e$ and $\phi=\pi$ (in Fig. 2 marked as $D$ - $D^{\prime}$ ), the reverse inductances may increase significantly,

$$
L_{J}^{-1}(n, 0, \pi) \approx \frac{(-1)^{n+1}}{4\left|j_{1}-j_{2}\right|} L_{J 0}^{-1}, \quad n=0 \text { and } 1,
$$

due to small asymmetry of the transistor parameters, $\left|j_{1}-j_{2}\right| \ll 1$. For example, in the case presented in

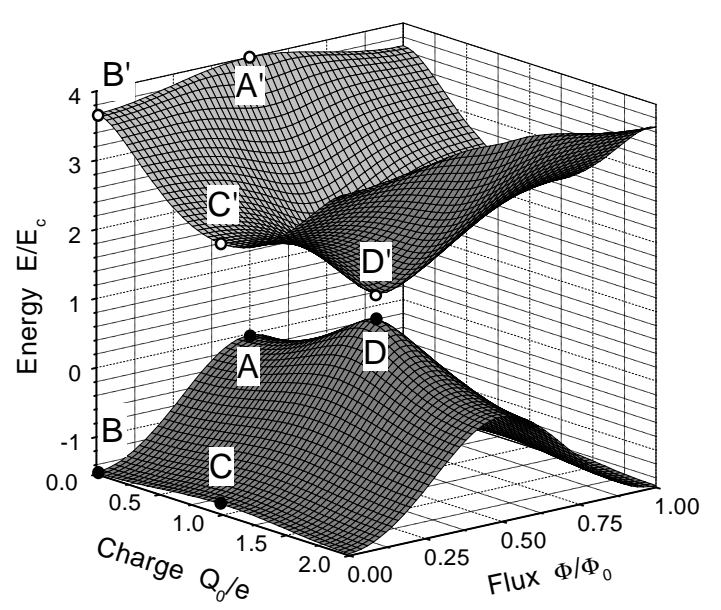

FIG. 2: Shape of energy bands $E_{0}$ and $E_{1}$ in the chargeflux qubit calculated for the mean Josephson coupling $E_{J 0} \equiv$ $\frac{1}{2}\left(E_{J 1}+E_{J 2}\right)=2 E_{c}$ and the Josephson coupling asymmetry parameter $\left|j_{1}-j_{2}\right|=\left(E_{J 1}-E_{J 2}\right) /\left(E_{J 1}+E_{J 2}\right)=0.1$. Black (hollow) circles on the zero (excited) band surface mark the locations of magic points $A\left(A^{\prime}\right), B\left(B^{\prime}\right)$, and $C\left(C^{\prime}\right)$ and the energy level avoided crossing point $D\left(D^{\prime}\right)$.
Fig. $2,\left|j_{1}-j_{2}\right|=0.1$ and $L_{J}^{-1}=\mp 2.5 L_{J 0}^{-1}$ for the zero and first band, respectively. In points $C$ and $C^{\prime}$, the absolute values $\left|L_{J}^{-1}\right|$ are smaller but the signs for $n=0$ and 1 are still different.

Coupling to the qubit causes a shift of the resonance frequency $\omega_{0}=\left(L_{T} C_{T}\right)^{-1 / 2}$ of the tank circuit, i.e., $\omega_{0}^{\prime}(n)=\omega_{0}+\delta \omega_{0}(n)$, where

$$
\delta \omega_{0}(n)=-\frac{1}{2} k^{2} \beta_{L} \frac{L_{J 0}}{L_{J}(n, q, \phi)} \omega_{0} .
$$

Here $k$ is the dimensionless coupling coefficient,

$$
k=\frac{M}{\sqrt{L_{T} L}}<1 .
$$

The resonance frequency shift $\delta \omega_{0}(n)$ carrying information about the qubit state $|n\rangle$ is found from the amplitude or/and phase of forced oscillations in the tank. For achieving sufficient resolution in such measurements, the quality factor of the tank circuit $Q$ should be about, or larger than, the ratio $\omega_{0} /\left|\delta \omega_{0}(0)-\delta \omega_{0}(1)\right|$.

\section{INHERENT AND EXTERNAL SOURCES OF DECOHERENCE}

In our consideration we had neglected the quasiparticle tunneling which inevitably causes dissipation of energy. Even rare tunneling of individual quasiparticles across the tunnel junctions, i.e., on and from the island, can decohere the qubit and completely destroy the readout regime described above. These processes lead to sudden change of the operation point, $q \rightarrow q \pm e$ and, possibly, the energy band index, i.e., cause relaxation $1 \rightarrow 0$.

The processes of single quasiparticle tunneling across a small Josephson junction had been studied by Averin and Likharev in Refs. 13, 14]. They had generalized the orthodox theory of single electron tunneling to the case of finite Josephson coupling, $E_{J} \neq 0$, taking into account the dynamics of essential phase factors $\exp ( \pm i \chi / 2)$ in the electron tunneling terms added to the Hamiltonian of type Eq. (2). These factors are the operators of singleelectron transfer and their nonzero matrix elements in our basis are

$$
e_{n n^{\prime}}^{ \pm}=\left\langle n, q|\exp ( \pm i \chi / 2)| q \pm e, n^{\prime}\right\rangle
$$

The rates of transitions $|q, n\rangle \rightarrow\left|q \pm e, n^{\prime}\right\rangle$ are given by

$$
\Gamma_{n n^{\prime}}^{ \pm}=\left|e_{n n^{\prime}}^{ \pm}\right|^{2} \frac{I_{\mathrm{qp}}\left(\epsilon_{n n^{\prime}}^{ \pm} / e\right)}{e}\left[1-\exp \left(-\frac{\epsilon_{n n^{\prime}}^{ \pm}}{k_{B} T}\right)\right]^{-1}
$$

In our case $I_{\mathrm{qp}}(U)$ is the quasiparticle current-voltage dependence of the network of two tunnel junctions of the qubit connected in parallel. Due to $2 e$-periodicity of the energy surfaces, the corresponding energy gains are identical,

$$
\epsilon_{n n^{\prime}}^{+}=\epsilon_{n n^{\prime}}^{-}=E_{n}(q, \phi)-E_{n^{\prime}}(q \pm e, \phi),
$$




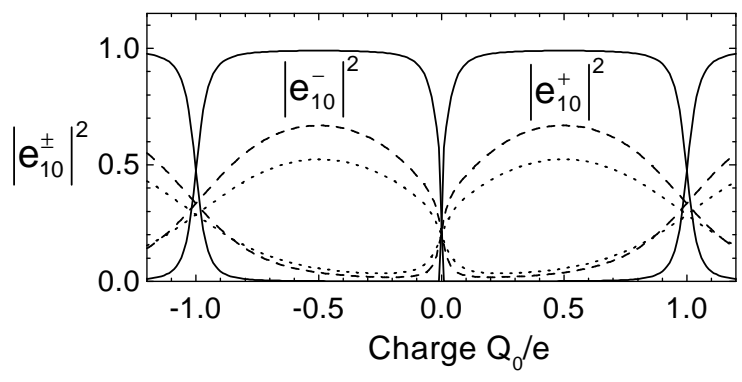

FIG. 3: Off-diagonal matrix elements of the single quasiparticle transfer operators $\exp ( \pm i \chi / 2)$ computed for different values of equivalent Josephson coupling set by flux $\Phi=\Phi_{0} / 2$ (solid lines), $\Phi_{0} / 4$ (dashed lines) and 0 (dotted lines). The qubit parameters are the same as in Fig. 2.

and their value depends on the operation point $\left\{Q_{0}, \Phi\right\}$ (see Fig. 2).

The relation between this energy and the superconductor energy gap $\Delta_{\mathrm{sc}}$ is important for having the quasiparticle transitions infrequent or even get rid of them. First, if voltage $U=\epsilon_{m n^{\prime}}^{ \pm} / e \leq 2 \Delta_{\mathrm{sc}} / e$, the quasiparticle current $I_{q p}(U)$ entering Eq. (17) is exponentially small, i.e., $\sim I_{c 0} \exp \left(-\Delta_{\mathrm{sc}} / k_{B} T\right)$ [15]. At larger voltages, $U>2 \Delta_{\mathrm{sc}} / e$, current $I_{q p}$ is enormously large, $\geq 2 I_{c 0}$. Therefore, in order to prevent intensive tunneling of quasiparticles the gain of energy, $\epsilon_{n n^{\prime}}^{ \pm}$, should never exceed $2 \Delta_{\mathrm{sc}}$. Secondly, if this gain is smaller than $\Delta_{\mathrm{sc}}$, then infrequent quasiparticle tunneling can, in principle, be blocked by the gap energy associated with one unpaired electron in the superconducting island (the socalled even-odd parity effect) [16].

Suppression of quasiparticle transitions within the zero energy band in superconducting $\mathrm{Al}$ single charge transistors and Cooper pair boxes was extensively investigated experimentally. Depending on experimental skill and luck (see, e.g., 17, 18, 19, 20]) the inspected devices often exhibited pure Cooper pair behavior when their charging energy $E_{c}$ was not larger than $\sim 100 \mu \mathrm{eV} \approx 0.5 \Delta_{\mathrm{Al}}$, where $\Delta_{\mathrm{Al}}$ is superconductor energy gap of aluminium. Since the energy gain for transitions in the Cooper pair boxes and low-voltage-biased transistors, $\epsilon_{00}^{ \pm}$, is less than $E_{c}$ for any $E_{J}$, the condition $E_{c}<\Delta_{\mathrm{sc}}$ can in a "good" qubit sample ensure suppression of quasiparticle tunneling in the ground state.

For quasiparticle transitions from the excited state this condition is clearly insufficient. For example, for small $E_{J}$ (corresponding to flux value $\Phi=\Phi_{0} / 2$, Eq. (3) ) the energy gain values are between about $E_{c}$ (for the process $D^{\prime} \rightarrow A$, see Fig. 2) and $4 E_{c}$ (for the processes $A^{\prime} \rightarrow D^{\prime}$ and $A^{\prime} \rightarrow D$ ). At larger $E_{J}$ both the minimum and maximum energy gain values are even larger. For example, for $E_{J}=4 E_{c}$ (i.e., $\Phi=0$ ), transitions $C^{\prime} \rightarrow B$ and $B^{\prime} \rightarrow C$ correspond to energy $\approx 4 E_{c}$ and $\approx 5 E_{c}$, respectively. Since the first multiplier factor in the expression for resulting relaxation rate,

$$
\left[\tau_{r}^{(\mathrm{qp})}\right]^{-1}=\Gamma_{10}^{+}+\Gamma_{10}^{-} \approx\left(\left|e_{10}^{+}\right|^{2}+\left|e_{10}^{-}\right|^{2}\right) \frac{I_{\mathrm{qp}}\left(\epsilon_{10}^{ \pm} / e\right)}{e},
$$

is nonzero for any $Q_{0}$ and $\Phi$ (see the plots of the two items in Fig. 31), only the condition $E_{c} \leq \Delta_{\mathrm{sc}} / 5$ can ensure suppression of these transitions in arbitrary operation point of our qubit. Possibly insufficiently small value of $E_{c}$ was the reason of very short relaxation time (tens of ns) in the recent experiment with a charge qubit by Duty et al. [20]. Their Al Cooper pair box had $E_{c} \approx 0.8 \Delta_{\mathrm{sc}}$ and $E_{J} \approx 0.4 E_{c}$, so the energy gain in the chosen operation point $\left(Q_{0}=0.4 e\right)$ was too large, i.e., about $2.2 E_{c} \approx 1.8 \Delta_{\mathrm{sc}}>\Delta_{\mathrm{sc}}$ (although in the ground state this sample nicely showed the pure Cooper pair characteristic).

Moreover, there are several sources of decoherence due to coupling of the qubit to the environmental degrees of freedom. For evaluating the effect of these sources on the qubit the coupling Hamiltonian term, $H_{\text {coupl }}=$ $H_{c}^{(e)}+H_{c}^{(m)}$, is included in the total Hamiltonian of the system,

$$
H=H_{0}+H_{\text {coupl }}+H_{\text {bath }}
$$

where $H_{\text {bath }}$ is a bath operator. $H_{c}^{(e)}$ and $H_{c}^{(m)}$ are the electric control line term and the magnetic coupling term respectively. The latter is associated with both the flux control line and the tank circuit. Fluctuations originating from the sources of gate- and flux-control lines may, in principle, lead to significant decoherence of the qubit. As was shown in Ref. 21] and demonstrated in experiments [3, 4, 5], these effects can, however, be minimized by choosing appropriate (minimum) coupling. On the other hand, decoherence caused by the tank-circuit-based readout system requires special analysis, because weakening of this coupling leads the input signal being reduced. Below we will start with sources of decoherence associated with the control lines and then will analyze the effect of the tank circuit and amplifier.

\section{COUPLING TO CHARGE CONTROL LINE}

The coupling of the charge-phase qubit to the electrical control line is actually similar to that of the gate coupling in the ordinary Cooper pair box 21]. However, here we assume that Josephson coupling parameter $\lambda$ is not necessarily small, as is usually assumed in the analysis of the charge qubits. This generalization of the model is essential because the external flux $\Phi_{m}$ changes the effective Josephson energy Eq. (3) of the qubit over a wide range. The assumption of not small $\lambda$ implies that the eigenstates of our system Eq. (5) are generally composed of several (not only two) plane-wave states. 
The coupling term can be presented as

$$
H_{c}^{(e)}=-2 e n \delta V_{e},
$$

where $\delta V_{e}$ is the operator of voltage fluctuations on the island in the absence of Josephson coupling. The charge operator is equal to $2 e n=Q_{0}-C \hat{V}$, so the essential part of the coupling Hamitonian is

$$
H_{c}^{(e)}=C \hat{V} \delta V_{e} .
$$

The voltage operator is equal to

$$
\hat{V}=\frac{\Phi_{0}}{2 \pi} \dot{\varphi}=\frac{\Phi_{0}}{2 \pi}\left(\dot{\chi}-\frac{\partial \gamma}{\partial \phi} \dot{\phi}\right)=\frac{\Phi_{0}}{2 \pi} \dot{\chi}
$$

Here we assumed slow variation of the total phase $\phi$, Eq. (9). The operator of voltage $\hat{V}$ is similar to the operator of velocity of an electron in the periodic electric potential of the crystal lattice 22], and its interband matrix elements are

$$
V_{n n^{\prime}}=\frac{\partial E_{n}}{\partial q} \delta_{n, n^{\prime}}+i \frac{E_{n}-E_{n^{\prime}}}{2 e} \chi_{n n^{\prime}}\left(1-\delta_{n, n^{\prime}}\right),
$$

where $\delta_{n, n^{\prime}}$ is the Kroneker delta and $\chi_{n n^{\prime}}$ are the matrix elements of the phase operator $\chi$ [1].

Finally, the coupling Hamiltonian, Eq. (22), takes the form

$$
H_{c}^{(e)}=\left(\sigma_{x} \sin \eta_{e}+\sigma_{z} \cos \eta_{e}\right) X_{e}
$$

where operator

$$
X_{e}=C\|V\| \delta V_{e}
$$

(a)

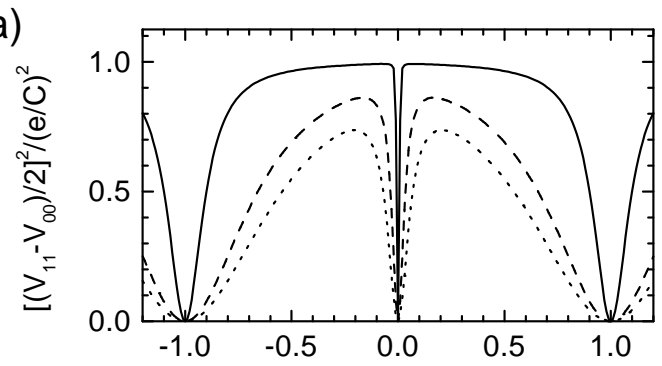

(b)

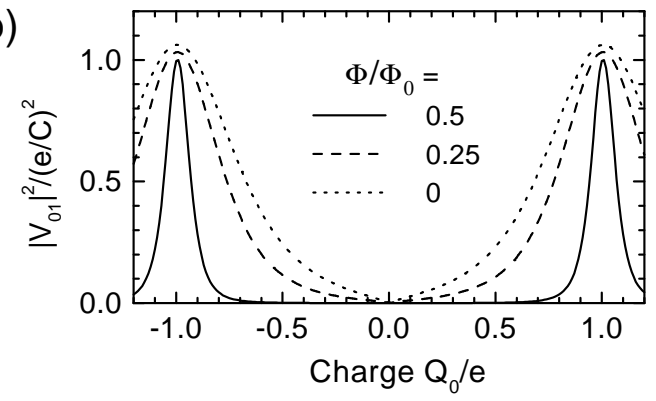

FIG. 4: The terms composed of diagonal (a) and off-diagonal (b) matrix elements of operator $\hat{V}$ and entering Eqs. (27) and (28) are presented for different values of flux $\Phi_{e}$ for the given qubit parameters (see caption of Fig. 2). with

$$
\|V\|=\frac{1}{2} \sqrt{\left(V_{11}-V_{00}\right)^{2}+4\left|V_{01}\right|^{2}}
$$

and

$$
\tan \eta_{e}=\frac{2\left|V_{01}\right|}{\left(V_{11}-V_{00}\right)}
$$

(The plots of the terms entering Eqs. (27) and (28) obtained by numerical calculations are presented in Fig. (4) Thus, $X_{e}=\sum_{a} C_{a} x_{a}$ can be considered as an operator of the bath 23] with the Hamiltonian

$$
H_{b}^{(e)}=\sum_{a}\left(\frac{p_{a}^{2}}{2 m_{a}}+\frac{m_{a} \omega_{a}^{2} x_{a}^{2}}{2}\right)
$$

and the spectral density $S_{X}^{(e)}(\omega)=C^{2}\|V\|^{2} S_{V}^{(e)}(\omega)=$ $J_{e}(\omega) \Theta(\omega, T) / \omega$. Here the oscillator energy function is

$$
\Theta(\omega, T)=\frac{\hbar \omega}{2} \operatorname{coth} \frac{\hbar \omega}{2 k_{B} T}
$$

and

$$
J_{e}(\omega)=\frac{\pi}{2} \sum_{a} \frac{C_{a}^{2}}{m_{a} \omega_{a}} \delta\left(\omega-\omega_{a}\right) .
$$

Assuming smallness of $C_{g}$, the spectral density $S_{V}^{(e)}$ of the fluctuations of $\delta V_{e}$ is given by

$$
S_{V}^{(e)}(\omega)=\frac{2}{\pi}\left(\frac{C_{g}}{C}\right)^{2} \operatorname{Re} Z_{t}(\omega) \Theta(\omega, T),
$$

where $Z_{t}=\left(i \omega C_{g}+Z_{g}^{-1}\right)^{-1}$ is determined by parallel connection of the qubit capacitance $C_{g} C /\left(C_{g}+C\right) \approx C_{g}$ and the gate line impedance $Z_{g}(\omega) \sim R_{100} \equiv 100 \Omega$. Therefore, for frequencies up to $\omega_{g} \equiv\left(R_{100} C_{g}\right)^{-1} \gg \epsilon / \hbar$, i.e., at all characteristic frequencies of the system, $\operatorname{Re} Z_{t}=$ $R_{100}$. This is the case of linear damping in the CaldeiraLeggett model,

$$
J_{e}(\omega)=\frac{\pi}{2} \alpha_{e} \hbar \omega
$$

with the dimensionless factor

$$
\alpha_{e}(q, \phi)=\left(\frac{C_{g}\|V\|}{e}\right)^{2} \frac{R_{100}}{R_{Q}} \lesssim\left(\frac{C_{g}}{C}\right)^{2} \frac{R_{100}}{R_{Q}},
$$

where $R_{Q}=h / 4 e^{2} \approx 6.45 \mathrm{k} \Omega$, the resistance quantum. The estimate similar to the last expression in Eq. (34), was given earlier in Ref. 21] for small $\lambda$.

Relaxation and dephasing caused by the charge control line can, therefore, be described by the spin-boson model with linear damping [24]. The corresponding rates are given by the expressions

$$
\left[\tau_{r}^{(e)}\right]^{-1}=\pi \alpha_{e} \sin ^{2} \eta_{e} \Omega \operatorname{coth} \frac{\hbar \Omega}{2 k_{B} T},
$$


and

$$
\left[\tau_{\varphi}^{(e)}\right]^{-1}=\left[2 \tau_{r}^{(e)}\right]^{-1}+\pi \alpha_{e} \cos ^{2} \eta_{e} \frac{2 k_{B} T}{\hbar} .
$$

One can see that in accordance with the conclusions of Refs. 21, 25], reducing the coupling coefficient $\alpha_{e}$ by a small factor $\left(C_{g} / C\right)^{2} \ll 1$ can significantly depress the decoherence rates.

\section{COUPLING TO FLUX CONTROL LINE}

The inductive coupling of the qubit loop with the control and readout circuits is described by the Hamiltonian

$$
H_{c}^{(m)}=-\hat{I}_{s}\left(\delta \Phi_{m}+\delta \Phi_{T}\right),
$$

where $\hat{I}_{s}$ is the operator of current circulating in the qubit loop, $\delta \Phi_{m}=M_{m} \delta I_{m}$, the bath operator (proportional to fluctuations of current $\delta I_{m}$ in the control inductance $\left.L_{m}\right) ; \delta \Phi_{T}=M \delta I$ is the operator of flux associated with current fluctuations in the tank circuit.

To specify the coupling, we present the operator $\hat{I}_{s}$ in the eigen basis (5), i.e., we find the matrix elements

$$
\left\langle n\left|\hat{I}_{s}\right| n^{\prime}\right\rangle, \quad n, n^{\prime}=0,1 .
$$

In the general case, $\hat{I}_{s}$ is given by the expression

$$
\hat{I}_{s}=\kappa_{1} \dot{Q}_{1}+\kappa_{2} \dot{Q}_{2}
$$

(a)

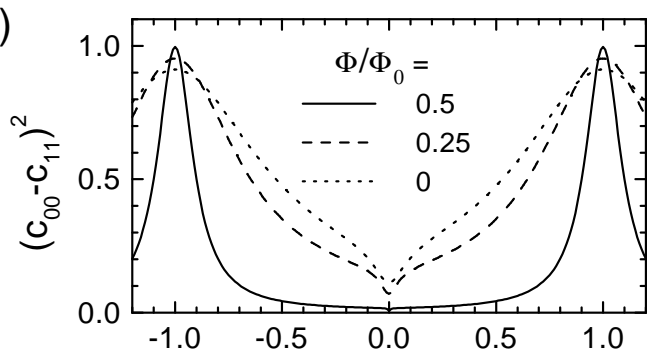

(b)

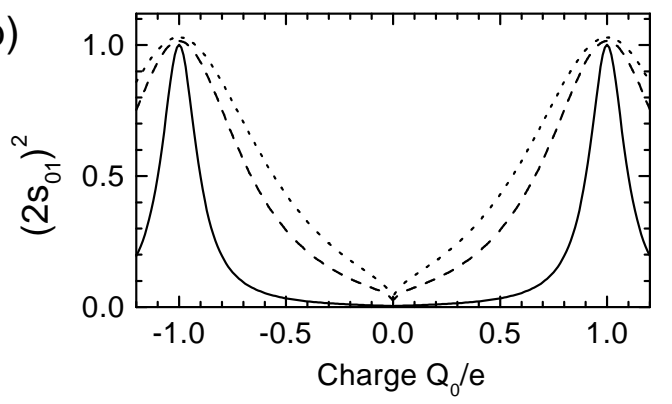

FIG. 5: Terms composed of diagonal (a) and off-diagonal (b) matrix elements of operators $\cos \chi$ and $\sin \chi$, respectively, calculated for different values of flux $\Phi_{e}$ for the given qubit parameters (see caption of Fig. 2). where the dimensionless factors $\kappa_{1,2}=C_{2,1} / C$ with the identity $\kappa_{1}+\kappa_{2}=1$. Quantities $Q_{1,2}=-2 e i \frac{\partial}{\partial \varphi_{1,2}}$ are the charges on the first and second junction, respectively, and their time derivatives are the Josephson supercurrents,

$$
\dot{Q}_{1,2}=\frac{i}{\hbar}\left[Q_{1,2}, H_{0}\right]=I_{c 1, c 2} \sin \varphi_{1,2} .
$$

Using identity $\varphi_{1,2}=\frac{\phi}{2} \pm \varphi=\frac{\phi}{2} \pm \chi \mp \gamma$ and Eq. (44), the circulating current can be presented as

$$
\hat{I}_{s}=I_{1}(\phi) \cos \chi+I_{2}(\phi) \sin \chi .
$$

The amplitudes of these two components are

$$
I_{1}=\frac{2 \pi}{\Phi_{0}} \frac{E_{J 1} E_{J 2}}{E_{J}(\phi)} \sin \phi,
$$

and

$$
\begin{aligned}
I_{2} & =\left(j_{1}-j_{2}\right)\left(\kappa_{1} j_{1}+\kappa_{2} j_{2}\right) \frac{8 \pi}{\Phi_{0}} \frac{E_{J 0}^{2}}{E_{J}(\phi)} \\
& +\left(\kappa_{1}-\kappa_{2}\right) \frac{4 \pi}{\Phi_{0}} \frac{E_{J 1} E_{J 2}}{E_{J}(\phi)} \cos ^{2} \frac{\phi}{2},
\end{aligned}
$$

respectively.

Since the Hamiltonian, Eq. (2), is an even function of $\chi$, the operators $\cos \chi$ and $\sin \chi$ entering Eq. (41) are diagonal and off-diagonal, respectively. Amplitude $I_{1}$ is merely the classical Josephson current across two largecapacitance junctions as a function of the overall phase difference $\phi$, while the diagonal term $\cos \chi$ describes the suppression of this current due to the charging effect $\left(E_{c} \neq 0\right)$ (see, for example, Ref. [26]). The second, offdiagonal, term in Eq. (41) is due to asymmetry of the transistor; it gives rise to the interband transitions $0 \leftrightarrow 1$. Denoting the nonzero values of the corresponding matrix elements as

$$
c_{00}=\langle 0|\cos \chi| 0\rangle, \quad c_{11}=\langle 1|\cos \chi| 1\rangle,
$$

and

$$
s_{01}=|\langle 0|\sin \chi| 1\rangle|,
$$

we obtain the coupling Hamiltonian Eq. (37) in the form

$$
H_{c}^{(m)}=\left(\sigma_{y} \sin \eta_{m}+\sigma_{z} \cos \eta_{m}\right)\left(X_{m}+X_{T}\right),
$$

where

$$
\begin{gathered}
X_{m, T}=-\|I\| \delta \Phi_{m, T}, \\
\|I\|=\sqrt{\left[\left(c_{11}-c_{00}\right) I_{1}\right]^{2}+\left[2 s_{01} I_{2}\right]^{2}}, \\
\tan \eta_{m}=\frac{2 s_{01} I_{2}}{\left(c_{11}-c_{00}\right) I_{1}},
\end{gathered}
$$


(see the plots of terms entering these expressions in Fig. (5).

First we omit in Eq. (46) the term $X_{T}$ associated with fluctuations of the tank circuit and focus on the effect of fluctuations in the flux control line $\delta \Phi_{m}=M_{m} \delta I_{m}$. Assuming real impedance of the flux control line, $Z_{m} \sim$ $R_{100}$, we obtain the spectral density of the operator $X_{m} \propto \delta I_{m}$ in the form $S_{X}^{(m)}(\omega)=M_{m}^{2}\|I\|^{2} S_{I}^{(m)}(\omega)=$ $J_{m}(\omega) \Theta(\omega, T) / \omega$. At frequencies below $\omega_{m} \equiv R_{100} / L_{m}$ the function $J_{m}$ is linear,

$$
J_{m}(\omega)=\frac{\pi}{2} \alpha_{m} \hbar \omega
$$

with the dimensionless coupling factor

$$
\alpha_{m}(q, \phi)=\left(\frac{2 M_{m}\|I\|}{\Phi_{0}}\right)^{2} \frac{R_{Q}}{R_{100}} .
$$

At higher frequencies, $\omega>\omega_{m}$, the effective damping decays as $\left(\omega_{m} / \omega\right)^{2}$.

In fact, Eq. (51) describes the effect of coupling to the control flux in the general case. An estimate of the coupling factor based on the evaluation

$$
\|I\| \approx \frac{1}{2}\left|\frac{\partial E_{J}}{\partial \Phi}\right|
$$

which is valid for symmetric transistor $\left(I_{2}=0\right)$ with small $E_{J}$, was made by Makhlin et al. 21]. Small mutual inductance $M_{m}$ [21, 25] leads to small $\alpha_{m}$ and, therefore, causes significant depression of the corresponding relaxation rate,

$$
\left[\tau_{r}^{(m)}\right]^{-1}=\pi \alpha_{m} \sin ^{2} \eta_{m} \Omega \operatorname{coth} \frac{\hbar \Omega}{2 k_{B} T}
$$

and dephasing rate,

$$
\left[\tau_{\phi}^{(m)}\right]^{-1}=\left[2 \tau_{r}^{(m)}\right]^{-1}+\pi \alpha_{m} \cos ^{2} \eta_{m} \frac{2 k_{B} T}{\hbar} .
$$

So far we have considered the effects of decoherence due to the charge and flux control lines as two independent effects. Actually, one has to describe them together using a multibath model 21]. If either of these decoherence effects is small, i.e. the so-called Hamiltoniandominated regime is realized, the total rates due to contributions of the two control lines are given by

$$
\begin{gathered}
{\left[\tau_{r}^{(c)}\right]^{-1}=\left[\tau_{r}^{(e)}\right]^{-1}+\left[\tau_{r}^{(m)}\right]^{-1},} \\
{\left[\tau_{\varphi}^{(c)}\right]^{-1}=\left[2 \tau_{r}^{(c)}\right]^{-1}+\left[\tau_{\varphi}^{(e)}\right]^{-1}+\left[\tau_{\varphi}^{(m)}\right]^{-1} .}
\end{gathered}
$$

In our model we shall assume that such a regime is realized and, moreover, the resulting rates, Eqs. (55) and (56), can be made negligibly small. Below we will focus on the effect of the readout circuit, whose coupling strength has to be optimized.

\section{DECOHERENCE DUE TO READOUT SYSTEM}

In contrast to the control lines, coupling to a readout device (in our case the tank circuit with amplifier) cannot be made arbitrarily small in order to reduce the decoherence. This coupling should ensure sufficiently strong signals at the amplifier input in order to perform a measurement with a reasonable signal-to-noise ratio on a time scale shorter than that determined by other factors, namely $\tau_{r}^{(c)}$. Moreover, without an efficient switch (see possible design of such a switch in, e.g., Ref. 27]), such a coupling may cause significant dephasing of the qubit during quantum gate manipulation.

Inductive qubit coupling to the tank circuit is described by the Hamiltonian Eq. (46). The spectral density of fluctuations of the corresponding variable $X_{T} \propto$ $\delta \Phi_{T}=M \delta I$ is expressed as

$$
S_{X}^{(T)}(\omega)=\frac{2}{\pi} M^{2}\|I\|^{2} S_{I}^{(T)}(\omega)=J_{T}(\omega) \frac{\Theta\left(\omega, T^{*}\right)}{\omega},
$$

where $S_{I}^{(T)}(\omega)$ is the spectral density of the noise current $\delta I$ across inductance $L_{T}$. Since the cold (superconducting) tank circuit itself presumably has very low losses, a backaction noise $\delta I$ of the amplifier is dominating. It is associated with input real impedance, modeled by $R_{p}$ or $R_{s}$ for parallel and serial configurations, respectively (see Fig. 1). $T^{*}$ is the effective temperature associated with this impedance.

Spectral density $S_{I}^{(T)}$ and function $J_{T}(\omega)$ can be found from a network consideration. Neglecting small detuning $\delta \omega_{0} \ll \omega_{0}$, in the case of the parallel network (Fig. 1b), the spectral function $J_{T}$ is given by the expression

$$
J_{T}^{(p)}(\omega)=\frac{2}{\pi} \alpha_{p} \hbar \omega \frac{\omega_{0}^{4}}{\left(\omega^{2}-\omega_{0}^{2}\right)^{2}+\omega^{2} \omega_{0}^{2} Q^{-2}},
$$

with

$$
\alpha_{p}=\left(\frac{2 M\|I\|}{\Phi_{0}}\right)^{2} \frac{R_{Q}}{R_{p}}=\frac{k^{2} \beta_{L}}{\pi Q} \frac{\|I\|^{2}}{e \omega_{0} I_{c 0}},
$$

where the quality factor $Q=\omega_{0} C_{T} R_{p}=R_{p} / \omega_{0} L_{T}$. In the case of the serial network shown in Fig. 1c,

$$
J_{T}^{(s)}(\omega)=\frac{2}{\pi} \alpha_{s} \hbar \omega \frac{\omega^{2} \omega_{0}^{2}}{\left(\omega^{2}-\omega_{0}^{2}\right)^{2}+\omega^{2} \omega_{0}^{2} Q^{-2}},
$$

with

$$
\alpha_{s}=\left(\frac{2 M\|I\|}{\Phi_{0}}\right)^{2} \frac{R_{Q} R_{s}}{\left(\omega_{0} L\right)^{2}}
$$

and $Q=\left(\omega_{0} C_{T} R_{s}\right)^{-1}=\omega_{0} L_{T} / R_{s}$.

In contrast to the linear spectral functions for the control lines, Eqs. (33) and (50), the functions given by Eqs. (58) and (60) describe a structured bath, viz. they both are of a Lorentzian (resonance) shape. A similar 
situation emerges, for example, in the case of the flux qubit with readout using a $C$-shunted dc SQUID 25]. The theoretical analysis of the spin-boson model with a structured bath was made by Kleff et al. 28] on the basis of the flow equations. If coupling is, as in our case, weak, only the high frequency $(\omega \sim \Omega)$ and low frequency $(\omega \rightarrow 0)$ behaviors of $J(\omega)$ account for relaxation and dephasing, respectively [25, 29, 30].

Since frequency $\Omega$ is typically about tens of $\mathrm{GHz}$ and the distance between qubit and amplifier presumably exceeds the wavelength, the effective real admittance of the parallel circuit at these frequencies is equal to $R_{100}^{-1}$ and the impedance of the serial circuit $\approx R_{100}$. Therefore, the relaxation rates increase by factors $g_{p}=R_{p} / R_{100} \gg 1$ and $g_{s}=R_{100} / R_{s} \gg 1$, respectively.

For the parallel tank circuit the relaxation and dephasing rates (presumably, $\ll \omega_{0}$ ) are equal to

$$
\left[\tau_{r}^{(p)}\right]^{-1}=\pi g_{p} \alpha_{p} \sin ^{2} \eta_{m}\left(\frac{\omega_{0}}{\Omega}\right)^{4} \Omega \operatorname{coth} \frac{\hbar \Omega}{2 k_{B} T^{*}},
$$

and

$$
\left[\tau_{\varphi}^{(p)}\right]^{-1}=\left[2 \tau_{r}^{(p)}\right]^{-1}+\pi \alpha_{p} \cos ^{2} \eta_{m} \frac{2 k_{B} T^{*}}{\hbar},
$$

respectively. The relaxation rate is dramatically suppressed due to the small frequency ratio, $\left(\omega_{0} / \Omega\right) \ll 1$. For the serial configuration, the corresponding rates are

$$
\begin{gathered}
{\left[\tau_{r}^{(s)}\right]^{-1}=\pi g_{s} \alpha_{s} \sin ^{2} \eta_{m}\left(\frac{\omega_{0}}{\Omega}\right)^{2} \Omega \operatorname{coth} \frac{\hbar \Omega}{2 k_{B} T^{*}},} \\
{\left[\tau_{\varphi}^{(s)}\right]^{-1}=\left[2 \tau_{r}^{(s)}\right]^{-1} .}
\end{gathered}
$$

The dephasing rate is determined by the rate of relaxation, because at low frequency, $\omega \ll \omega_{0}$, function $J_{T}^{(s)}(\omega) \propto \omega^{3}$ [24]. Due to weaker decay of the serial circuit impedance at high frequencies, $\omega \gg \omega_{0}$, the relaxation rate is, however, substantially higher than that in the case of the parallel circuit configuration. We shall therefore focus our further consideration only on the parallel tank circuit as the more favorable (allowing longer measuring time).

\section{MAGIC POINTS AND SOME ESTIMATIONS}

The analysis of the coupling between the qubit and the tank circuit, Eqs. (46)-(49) and Fig. (5), shows that its strength $X_{T} \propto\|I\|$ and mixing angle $\eta_{m}$ can be significantly varied by choosing an appropriate operation point. For example, as can be seen from Eq. (42), the diagonal component of $X_{T}\left(\propto I_{1}\right)$ which essentially cause pure dephasing of the qubit, is zero, i.e., mixing angle $\eta_{m}=\pi / 2$, at phase values $\phi=0$ and $\pi$. The derivatives $\frac{\partial E_{0,1}}{\partial \phi}$ and, therefore, the circulating supercurrent are zero. Moreover, as illustrated in Fig. 5b, if the gate charge $Q_{0} \approx 0$ (i.e., derivatives $\frac{\partial E_{0,1}}{\partial Q_{0}}=0$ ), then $\left|s_{01}\right|$ and, hence, $X_{T}$ are minimum. In particular, at the bias flux $\Phi_{m}=\Phi_{0} / 2$ or, equivalently, $\phi=\pi$ (this point is marked in Fig. 2 as $A), E_{J}(\phi)=\left|E_{J 1}-E_{J 2}\right| \ll E_{c}$, so one can use the explicit expressions for the wave functions, Eqs. (A11) and (A12) of Ref. [11], and obtain

$$
\left|s_{01}\right|=\frac{1}{16 \sqrt{2}} \frac{E_{J}(\phi)}{E_{c}}=\frac{\left|j_{1}-j_{2}\right|}{8 \sqrt{2}} \frac{E_{J 0}}{E_{c}} .
$$

Then the value of $\|I\|$ (48) is

$$
\|I\|_{A}=2\left|s_{01}\right| I_{2} \approx \frac{\left|j_{1}-j_{2}\right|}{4 \sqrt{2}} \frac{E_{J 0}}{E_{c}} I_{c 0},
$$

where we have taken into account that $\kappa_{1} \sim \kappa_{2} \sim 0.5$ and the second term in Eq. (43), $\propto\left(\kappa_{1}-\kappa_{2}\right)$, vanishes due to the relation $\cos (\phi / 2)=0$. In the point $Q_{0}=0, \phi=0$ (marked as $B$ in Fig. 2), the Josephson energy $E_{J}(\phi)=2 E_{J 0}$ and $2\left|s_{01}\right|$ is approximately equal to $(1 / 8 \sqrt{2}) E_{J 0} / E_{c}$, so

$$
\|I\|_{B} \approx \frac{\left|j_{1}-j_{2}+\kappa_{1}-\kappa_{2}\right|}{8 \sqrt{2}} \frac{E_{J 0}}{E_{c}} I_{c 0},
$$

while for $Q_{0}=e$ (point $C$ in Fig. 2), $\left|s_{01}\right| \approx 0.5$ and

$$
\|I\|_{C} \approx\left|j_{1}-j_{2}+\kappa_{1}-\kappa_{2}\right| I_{c 0} .
$$

It is remarkable that the effect of asymmetry in critical currents and capacitances of the junctions can, in principle, cancel if $\left(j_{1}-j_{2}\right)=-\left(\kappa_{1}-\kappa_{2}\right)$. In practice, however, the signs of $\left(j_{1}-j_{2}\right)$ and $\left(\kappa_{1}-\kappa_{2}\right)$ normally are similar because the critical current and capacitance are both proportional to the junction area and such cancelling does not occur.

Comparing Eqs. (67), 687) and (69) one can see that on the assumption of small asymmetry of the transistor, $j_{1} \approx j_{2} \approx \kappa_{1} \approx \kappa_{2} \approx 0.5$, the coupling strength $\alpha_{p}$ in points $A\left(Q_{0}=0, \phi=\pi\right), B\left(Q_{0}=0, \phi=0\right)$ and $C\left(Q_{0}=e, \phi=0\right)$ is rather small, but it is significant in the point, $D\left(Q_{0}=e, \phi=\pi\right)$, where parameter $\left|s_{01}\right| \approx$ 0.5 and

$$
\|I\|_{D} \approx I_{c 0} .
$$

To illustrate this behavior, the coupling strength evaluated for typical parameters of the system is presented in Table 1.

From the point of view of operation with a minimum dephasing rate, the "magic" points $A, B$ and $C$, in which supercurrent $I_{1}=0$ (see Eq. (42)), are clearly preferable to other points in the $Q_{0}-\Phi$ plane. Therefore, manipulation of the qubit can, in principle, be performed in any of these points. The estimated values of the corresponding quality factor for quantum manipulation, $Q_{\varphi} \equiv \Omega \tau_{\varphi}^{(p)}$, 
TABLE I: Evaluated qubit parameters derived on the assumption $E_{J 0}=2 E_{c}=80 \mu \mathrm{eV}$ (i.e., $I_{c 0} \approx 40 \mathrm{nA}$ and $5 E_{c}=\Delta_{\mathrm{Al}} \approx$ $200 \mu \mathrm{eV}$, the energy gap of $\mathrm{Al}$ ) and $j_{1}-j_{2}=\kappa_{1}-\kappa_{2}=0.1$. The tank circuit quality factor $Q=100$, frequency $\omega_{0}=$ $2 \pi \times 100 \mathrm{MHz},\left(L_{T} / C_{T}\right)^{1 / 2}=100 \Omega, k^{2} Q \beta_{L}=20$ and temperature $T^{*}=1 \mathrm{~K} \gg T \sim 20 \mathrm{mK}$. As long as the dephasing rate in the magic points is nominally zero, a $0.1 \%$ inaccuracy of the adjustment of the values $\phi=\pi$ and 0 was assumed.

\begin{tabular}{ccccc}
\hline \hline Operation point: & $A-A^{\prime}$ & $B-B^{\prime}$ & $C-C^{\prime}$ & $D-D^{\prime}$ \\
\hline Frequency $\Omega / 2 \pi[\mathrm{GHz}]$ & 39 & 50 & 36 & 4 \\
Coupling strength $\alpha_{p}$ & $210^{-2}$ & $10^{-2}$ & $410^{-2}$ & 1 \\
Qubit quality factor $Q_{\varphi}$ & $310^{4}$ & $210^{5}$ & $10^{4}$ & $<30$ \\
Relaxation time $\tau_{r}^{(p)}[\mathrm{s}]$ & $810^{-2}$ & $10^{-1}$ & $610^{-3}$ & $10^{-7}$ \\
\hline \hline
\end{tabular}

given in Table 1, are sufficiently high. For example, in the case of preparation of the qubit in point $A$, the manipulation can be performed by means of a dc pulse applied to the transistor gate [3, 4, 20]. This pulse (with short rise and fall times) can rapidly switch the qubit, for example, to point $D$ and back to $A$ causing its evolution (although with significant dephasing) during the pulse span. Our qubit prepared in the ground state in point $A$ or $B$ or $C$ can be (preferably) manipulated by a pulse of microwave frequency, $\sim \Omega$, applied to the gate. For example, the Quantronium qubit in the experiment by Vion et al. [5] was manipulated by microwave pulses in point $C$.

For reading out the final state, the qubit dephasing is of minor importance, while the requirement of a sufficiently long relaxation time is decisive. Moreover, the relaxation rate may somewhat increase due to oscillations in the tank induced by a drive pulse (see Fig.6), which leads to the development of oscillations around a magic point along $\phi$ axis, Eq. (9). If the frequency of these oscillations is sufficiently low, $\omega_{\text {rf }} \ll \Omega$, they result only in a slow modulation of transition frequency $\Omega$. Increase in amplitude of steady oscillations up to $\phi_{a} \approx \pi / 2$ (determined by the amplitude of the drive pulse and detuning) yields a large output signal and still ensures the required resolution in the measurement provided the product $k^{2} Q \beta_{L}>1$ is sufficiently large. (At larger amplitudes $\phi_{a}$, the circuit operates in a non-linear regime probing an averaged reverse inductance of the qubit whose value, as well as the produced frequency shift $\delta \omega_{0}$, is smaller 31].) As points $A$ and $B$ lie on the axis $Q_{0}=0$ and are both characterized by a sufficiently long relaxation time, reading-out of the qubit state with the rf oscillation span $\pm \pi / 2$ is preferable in either point. In the case of operation point $C$, the limited amplitude of the oscillations does not reduce much the relaxation time either. Significant reduction of the relaxation time occurs in the vicinity of point $D$. Due to this property which is due to the dependence of the transversal coupling strength on $\phi$, Eqs. (43)-(49), the measurement of the Quantronium state using a switching current technique was possible in the middle of segment $C D$ (see Fig. 2), where the maxi- mum values of the circulating current in the excited and ground states were of different sign [5].

In the vicinity of energy level avoided crossing point $D$, in which the gap between the zero and first excited states is minimum, $\hbar \Omega=2\left|j_{1}-j_{2}\right| E_{J 0}$, oscillations of $\phi$ may cause Landau-Zener transitions $|0\rangle \leftrightarrow|1\rangle$ [32]. Probability of a such transition per single sweep

$$
p_{\mathrm{LZ}}=\exp \left[-2 \pi \frac{\left(j_{1}-j_{2}\right)^{2} E_{J 0}}{\phi_{a} \hbar \omega_{\mathrm{rf}}}\right],
$$

can be appreciable in a sufficiently symmetric transistor and/or at high driving frequency $\omega_{\text {rf }}$, i.e., when $\left|j_{1}-j_{2}\right| \lesssim\left(\hbar \omega_{\mathrm{rf}} / E_{J 0}\right)^{1 / 2}$. These transitions lead to unwanted mixing of the qubit states 33. In the vicinity of point $A^{\prime}$, where the gap between the first and second (not shown in Fig. 2) energy bands is smaller [9], $\hbar \Omega_{12}=\left(j_{1}-j_{2}\right)^{2} E_{J 0}^{2} / 2 E_{c}$, the Landau-Zener transitions $|1\rangle \leftrightarrow|2\rangle$ are more probable. Fortunately, the second energy band has the positive curvature, $\frac{\partial^{2} E_{2}(0, \phi=\pi)}{\partial \phi^{2}}>0$, so the mixing of these states might even improve the distinguishability of signals from the ground and excited states. More rigorous analysis of this effect on operation of the qubit in point $A$ is, however, needed.

Finally, let us evaluate the time of measurement required for the resolution of the states $n=0$ and $n=1$ in the most favorable magic points $A$ and $B$. As schematically shown in Fig. 6, an $\mathrm{rf}$ drive pulse is applied to the tank circuit just after manipulation of the qubit $(t=0)$ and induces growing oscillations in the tank. The amplitude of the oscillations of voltage $V$ approaches a steady value $A_{0}\left(A_{1}\right)$ for $n=0(n=1)$ after the time $t_{\text {rise }} \approx 2 \pi Q / \omega_{0}$. Assuming a corresponding amplitude of oscillations of phase $\phi_{a}=\pi / 2$, we obtain for the parameters of Table 1 the amplitudes

$$
A_{0}=\phi_{a} \frac{\Phi_{0}}{2 \pi} \frac{\omega L_{T}}{M}=\left(\frac{\pi \Phi_{0} \omega R_{p} I_{c 0}}{8 k^{2} Q \beta_{L}}\right)^{1 / 2} \approx 3 \mu \mathrm{V}
$$

and $A_{1} \approx 1 \mu \mathrm{V}$.

Assuming that the equivalent noise of a semiconductorbased amplifier referred to the input is of the order of Johnson voltage noise across $R_{p} \approx 10 \mathrm{k} \Omega$ at ambient temperature $T^{*} \sim 2 \mathrm{~K}$, i.e., $S_{V}^{1 / 2} \approx 1 \mathrm{nV} / \sqrt{\mathrm{Hz}}$, we can express the signal-to-noise ratio as

$$
\mathrm{SNR}=\frac{\left(A_{0}-A_{1}\right) \sqrt{t_{\mathrm{meas}}}}{S_{V}^{1 / 2}} \approx 210^{3} \sqrt{t_{\mathrm{meas}} / 1 \mathrm{~s}}
$$

where $t_{\text {meas }}$ is the time of measurement. This time should clearly be much shorter than the relaxation time $\tau_{r}^{(p)}$ (evaluated as $\approx 0.1 \mathrm{~s}$, see Table 1 ) and exceed the rise time of the oscillations in the tank $t_{\text {rise }} \approx 1 \mu \mathrm{s}$ (the latter condition nicely agrees with the requirement SNR $>1$ ). Thus, a drive pulse duration of $\sim 10 \mu$ s ensuring $t_{\text {meas }} \sim 10 \mu$ s seems to be a good choice as it yields the sufficiently high value of $\mathrm{SNR} \approx 6$. The latter (as well 
(a)

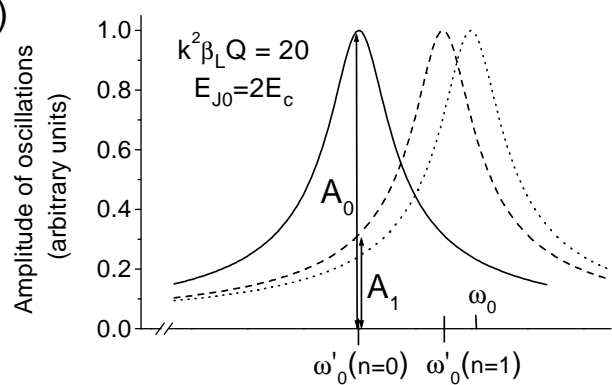

(b)

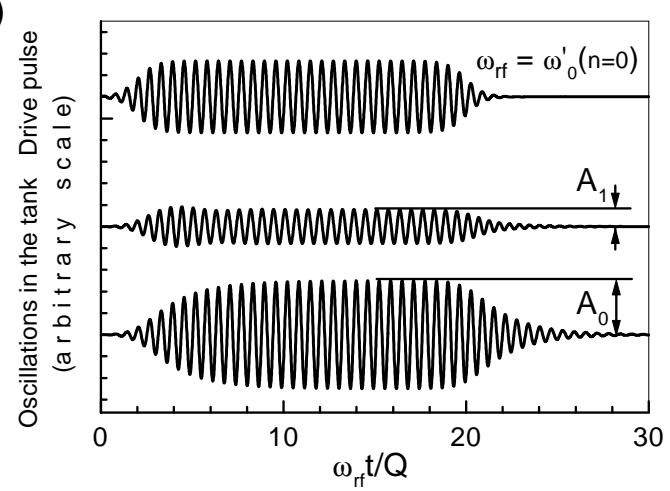

FIG. 6: The principle of narrow-band radio-frequency readout of the qubit. (a) The resonance curves of the uncoupled (dotted line) and coupled to the qubit tank circuit biased at operation point $A$ in the excited state (dashed line) and in the ground state (solid line). (b) Driving pulse applied to the tank circuit (top curve) and the response signal of the tank in resonance (the ground qubit state, bottom curve) and offresonance (excited state, middle curve). A smooth envelope of the driving pulse is used to suppress transient oscillations and has a small effect on the rise time of the response signal. For clarity the curves are shifted vertically.

as the quantum quality factor $Q_{\varphi}$ ) can be substantially improved by using a SQUID-based low-noise amplifier 34].

\section{CONCLUSION}

We have demonstrated that both manipulation and readout of the charge-phase qubit coupled to a tank circuit is, in principle, possible. More specifically, the decoherence effect of the electric and magnetic control lines can seemingly be minimized by reducing coupling to the qubit. The readout system based on the parallel tank circuit and cold amplifier can ensure sufficiently weak dephasing in the regime without $\mathrm{rf}$ drive. The dephasing rate strongly depends on the accuracy of adjusting the offset flux bias $\Phi_{m}=0$ or $\Phi_{m}=\Phi_{0} / 2$ corresponding to operation in the magic points. High symmetry of the Josephson junction parameters may further improve the coherence characteristics of the qubit. Since the $L C$

resonance tank circuit introduces only small noise at the high transition frequency of the qubit, $\Omega \gg \omega_{0}$, the rate of relaxation can also be made sufficiently small. Applying an rf drive pulse of limited span allows a readout of the qubit state in the regimes of single and repeated measurements.

Other problems in engineering Josephson qubits with weak decoherence are the $1 / f$ noise of critical currents of Josephson junctions 35 and the $1 / f$ background noise coupled to the charge variable [36] which have not been addressed in this paper but are equally important. Hopefully, in the given system these effects might not be as strong as in "traditional" tunnel-junction devices like dc SQUIDs and single electron transistors operating at nonzero voltage bias. Due to perfect decoupling of the superconducting loop with the single charge transistor from dc bias lines and due to the coherent nature of the Josephson current in the zero voltage bias regime, one could expect a minor backaction effect of the zerobias operating transistor on its critical current noise and charge noise which dramatically depend on the current fed (see, for example, Ref. [37]).

\section{ACKNOWLEDGEMENTS}

The author would like to thank Per Delsing, Yuriy Makhlin and Frank Wilhelm for stimulating discussions. This work was partially supported by the European Union through the SQUBIT-2 project.

* Electronic address: alexander.zorin@ptb.de

[1] See, for example, J. Clarke, Science 299, 1850 (2003) and references therein.

[2] V. Bouchiat, D. Vion, P. Joyez, D. Esteve, and M. Devoret, Phys. Scripta T76, 165 (1998).

[3] Y. Nakamura, Yu. A. Pashkin and J. S. Tsai, Nature 398, 768 (1999); Yu. A. Pashkin, T. Yamamoto, O. Astafiev, Y. Nakamura, D. V. Averin and J. S. Tsai, Nature 421, 823 (2003).

[4] K. Bladh, D. Gunnarson, G. Johansson, A. Käck, G. Wendin, A. Aassime, M. Taslakov and P. Delsing, Phys. Scripta T102, 167 (2002).

[5] D. Vion, A. Aassime, A. Cottet, P. Joyez, H. Pothier, C. Urbina, D. Esteve and M. H. Devoret, Science 296, 886 (2002).

[6] A. B. Zorin, Physica C 368, 284 (2002).

[7] R. Rifkin and B. S. Deaver, Jr., Phys. Rev. B 13, 3894 (1976).

[8] D. V. Averin, Phys. Rev. Lett. 88, 207901 (2002).

[9] Handbook of Mathematical Functions, edited by M. Abramowitz and I. A. Stegun (U.S. GPO, Washingtin, D.C., 1972), Chapter 20.

[10] D. V. Averin, A. B. Zorin, and K. K. Likharev, Sov. Phys. JETP 88, 697 (1985). 
[11] K. K. Likharev and A. B. Zorin, J. Low Temp. Phys. 59, 347 (1985).

[12] In fact, the energy spectrum of this system is similar to that of Quantronium [5], having one additional large Josephson junction in the loop.

[13] D. V. Averin, Fiz. Nizk. Temp. 13, 364 (1987) [Sov. J. Low Temp. 13, 208 (1987)].

[14] D. V. Averin and K. K. Likharev, in Mesoscopic Phenomena in Solids, edited by B. L. Altshuler, P. A. Lee, and R. A. Webb (Elsevier, Amsterdam, 1991), p.175.

[15] See, for example, a simple approximation formula in S. Ramo, J. R. Whinnery and T. van Duzer, Fields and Waves in Communication Electronics (John Willey and Sons, New York, 1965), p. 211.

[16] As follows from the entropy consideration, for finite volume of island and nonzero temperature the threshold value is somewhat smaller than $\Delta_{\mathrm{sc}}$, see, for details M. T. Tuominen, J. M. Hergenrother, T.S. Tighe and M. Tinkham, Phys. Rev. Lett. 69, 1997 (1992).

[17] P. Joyez, P. Lafarge, A. Filipe, D. Esteve, C. Urbina, and M. H. Devoret, Phys. Rev. Lett. 72, 2458 (1994).

[18] A. Amar, D. Song, C. J. Lobb, and F. C. Wellstood, Phys. Rev. Lett. 72, 3234 (1994).

[19] J. Männik and J. E. Lukens, Preprint (2003), available at http://arxiv.org/abs/cond-mat/0305190.

[20] T. Duty, D. Gunnarsson, K. Bladh, R. J. Schoelkopf and P. Delsing, Preprint (2003), available at http://arxiv.org/abs/cond-mat/0305433.

[21] Yu. Makhlin, G. Schön, and A. Shnirman, Rev. Mod. Phys. 73, 357 (2001).

[22] E. M. Lifshitz and L.P. Pitaevskii, Statistical Physics, Part 2 (Pergamon Press, Oxford, 1980).

[23] A. O. Cladeira and A. J. Leggett, Ann. Phys. 149, 374 (1983).

[24] A. J. Leggett, S. Chakravarty, A. T. Dorsey, M.P.A. Fisher, A. Garg and W. Zwerger, Rev.
Mod. Phys. 59, 1 (1987).

[25] C. H. van der Wal, F. K. Wilhelm, C. J. P. M. Harmans and J. E. Mooij, Eur. Phys. J. B 31, 111 (2003).

[26] A. B. Zorin, Phys. Rev. Lett. 76, 4408 (1996).

[27] J. Clarke, T. L. Robertson, B. L. T. Plourde, A. GarcíaMartinez, P.A. Reichardt, D. J. van Harlingen, B. Chesca, R. Kleiner, Y. Makhlin, G. Schön, A. Shnirman and F.K. Wilhelm, Phys. Scripta T102, 173 (2002).

[28] S. Kleff, S. Kehrein and J.von Delft, Preprint (2003), available at http://arxiv.org/abs/cond-mat/0304177.

[29] M. Grifoni, E. Paladino and U. Weiss, Eur. Phys. J. B 10, 719 (1999).

[30] L. Tian, S. Lloyd and T. P. Orlando, Phys. Rev. B 65, 144516 (2002).

[31] A. B. Zorin, Phys. Rev. Lett. 86, 3388 (2001).

[32] L. D. Landau, Phys. Z. Sowjetunion 1, 89 (1932); C. Zener, Proc. R. Soc. London, Ser. A 137, 696 (1932).

[33] A. V. Shytov, D. A. Ivanov and M. V. Feigel'man, Preprint (2001), available at http://arxiv.org/abs/condmat/0110490.

[34] M.-O. André, M. Mück, J. Clarke, J. Gail, and C. Heiden, Appl. Phys. Lett. 75, 698 (1999); M. Mück, J. B. Kycia, and J. Clarke, Appl. Phys. Lett. 78, 967 (2001).

[35] D. J. van Harlingen, Talk at NATO Advanced Research Workshop "Coherent charge and spin transport on a nanoscale" (June 8-12, 2003, Chernogolovka, Moscow Region, Russia), available at http://www.itp.ac.ru/ meso03/pdf/van_harlingen.pdf.

[36] E. Paladino, L. Faoro, G. Falci, and R. Fazio, Phys. Rev. Lett. 88, 228304 (2002).

[37] V.A. Krupenin, D. E. Presnov, M. N. Savvateev, H. Scherer, A. B. Zorin and J. Niemeyer, J. Appl. Phys. 84, 3212 (1998). 